Research Article

\title{
Study on the Effect of Surface Roughness on the Spectral Unmixing of Mixed Pixels
}

\author{
Haonan Zhang $\mathbb{D}^{1,2}$ Xingping Wen $\mathbb{D}^{1,},{ }^{1,2}$ Junlong Xu, ${ }^{1,2}$ Dayou Luo, ${ }^{1,2}$ and Ping He ${ }^{1,2,3}$ \\ ${ }^{1}$ Faculty of Land Resource Engineering, Kunming University of Science and Technology, Kunming 650093, China \\ ${ }^{2}$ Mineral Resources Prediction and Evaluation Engineering Laboratory of Yunnan Province, Kunming 650093, China \\ ${ }^{3}$ School of Fine Art and Design, Kunming University, Kunming 650000, China \\ Correspondence should be addressed to Xingping Wen; wfxyp@qq.com
}

Received 18 March 2020; Revised 23 May 2020; Accepted 5 June 2020; Published 8 July 2020

Academic Editor: Luciano Bachmann

Copyright (c) 2020 Haonan Zhang et al. This is an open access article distributed under the Creative Commons Attribution License, which permits unrestricted use, distribution, and reproduction in any medium, provided the original work is properly cited.

In the spectrum measurement experiment, the roughness of the object surface is an essential factor that cannot be ignored. In this experiment, a group of mixed pixel samples with different mixing ratios were designed, and these samples were printed on four kinds of papers with different roughness. The spectral characteristics of mixed pixels with different roughness are quantitatively analyzed by using the measured spectral data. The linear spectral mixture model is used for spectral decomposition, and the effect of roughness on the unmixing precision of mixed pixels was studied. The surface roughness will affect the reflectivity of the mixed pixel. Specifically, the higher the roughness is, the higher the reflectivity of the sample is. This phenomenon is more noticeable when the proportion of white endmember $(P W E)$ is large, and as the white area ratio decreases, the reflectance difference gradually decreases. When the surface roughness of the sample is less than $3.339 \mu \mathrm{m}$, the spectral decomposition is performed using a linear spectral mixing model in the visible light band. The average error of the unmixing is less than $0.53 \%$, which is lower than the conventional standard spectral measurement error. In other words, when the surface roughness of the sample is controlled within a specific range, the effect of roughness on the unmixing accuracy of the mixed pixels is small, and this effect can be almost ignored. Multiple scattering within the pixels is the key to model selection and unmixing accuracy, when using the ASD FieldSpec3 spectrometer to perform spectral reflectance measurement and linear spectral unmixing experiments. If the surface roughness of the sample to be measured is less than the maximum wavelength of the spectrometer, the experimental results believe that the photon energy is mainly mirror reflection on the surface of the object and diffuse reflection. At this time, it is still a better choice to use a linear spectral mixing model to decompose the mixed pixels.

\section{Introduction}

The information recorded by each pixel in the remote sensing image is the sum of the radiant energy of all target scenes in the ground range corresponding to the instantaneous field of view angle of the detection unit; such a pixel is called a mixed pixel $[1,2]$. Whether it is aerial remote sensing or aerospace remote sensing, the spectral information obtained by the sensor is a mixed spectrum containing multiple target scenes, so almost all pixels in remote sensing observations are mixed pixels [3]. There are three main reasons for mixed pixels. First is the composition of the detection target itself: the influence of geometric structure, position distribution, and other factors, resulting in the generation of mixed pixels similar to a specific type of scene [4]. Second reason is due to the different observation scale of the sensor, resulting in different spatial resolution of the image [5]. Third, this situation is caused by the background influence of the detection target, such as mixed pixels caused by target shadows, ground fluctuations, and atmospheric scattering [6]. Because the above reasons are unavoidable in actual remote sensing observations, these factors are indispensable when conducting mixed pixel spectral measurement experiments should all be considered.

The surface of most objects in nature is a rough surface relative to visible wavelengths. The surface of most objects is a rough surface that radiates relatively to visible wavelengths [7]. When the radiant brightness of an object's surface does 
not change with the angle of view, the object is called a Lambert reflector [8]. However, the surface of the object has anisotropic characteristics for the scattering of solar shortwave radiation, and almost all objects have non-Lambertian features. The reflection of non-Lambert reflector surfaces is between diffuse reflection and specular reflection. There are multiangle and multidirectional spatial distribution characteristics $[9,10]$. The surface roughness of objects is one of the main factors affecting emission, scattering, and radiation, which is essential for the research and application of passive microwave remote sensing. In the visible light and near-infrared band, roughness is one of the essential structural parameters on the surface of the scene, which directly affects the distribution characteristics of the target's retro reflection [11]. The phenomenon of visible light reflected by the surface of the object has two types, specular reflection and diffuse reflection, which is usually related to the roughness of the surface. When the roughness of the surface is less than the wavelength of the human wave, specular reflection is formed. At this time, the reflection angle is equal to the human angle. The highly polished metal plate is secularly reflected [12-14]. Diffuse reflection is formed when the uneven surface size is larger than the wavelength of the incident wave, and the reflection line is very irregular. For example, the heating furnace tube, the refractory brick surface, and so on are mostly diffuse reflection $[15,16]$. The spectral reflectance of a mixed pixel not only depends on the internal determining factors, such as endmember composition and composition structure, but also is influenced by external observation conditions factors such as observation azimuth, observation distance, atmospheric pressure, temperature, humidity, and meteorological conditions. In addition to the above factors, the influence of the surface roughness of the mixed pixels cannot be neglected. Regarding the effect of degree, especially when the roughness of the sample surface is substantially different, roughness is an essential factor that affects the spectral characteristics and the effect of spectral unmixing. Xie et al. used the atomic force scanning probe microscope to obtain the surface roughness data of granite. Taking into account the incident shielding and multiple scattering effects in the surface scattering process, the bidirectional reflection distribution function of the actual surface was simulated [17]. Based on the field-measured multiangle hyperspectral reflection information, $\mathrm{Lu}$ and Zhao analyzed the influence of surface roughness variation on the reflection information distribution of sandy land and used the model parameters to invert the roughness characteristics [18]. Kang et al. use multiangle observation information to soil surface roughness, exploring a new method for estimating surface parameters by combining optical and microwave remote sensing data. In order to simulate the distribution characteristics of the surface reflection of soil surface pixels under different roughness conditions, a geometric optical model of bare soil surface and reflection is established [19].

In terms of mixed pixel spectral unmixing, researchers have proposed a large number of mixed pixel decomposition models, mainly based on linear spectral mixing models and nonlinear spectral mixing models [20]. Among them, the linear spectral mixing model regards the mixed pixel spectrum. It is an area-weighted linear combination of component spectra within a pixel. The model believes that light does not interact between pixel components. The mixed spectrum is formed after the spectrum of each pixel component enters the sensor. A large number of laboratory and field experiments show that, in the case of uniform illumination and a smooth surface, the signal received by the detector is mainly a single scattering, and the linear spectral mixture model has a better decomposition effect. Because the linear spectral mixing model is more close to remote sensing transmission theory and convenient and practical, it has been widely used in the study of mixed pixel decomposition [21, 22]. However, it is affected by factors such as atmospheric scattering, topographic fluctuations, and target surface roughness. In these cases, the multiple scattering effects between the internal components of the pixel are enhanced. At this time, the nonlinear spectral mixing model is more in line with the actual situation, so it is particularly important to choose different models according to the actual situation [23]. In terms of model decomposition accuracy, Wang and others compared the effects of linear spectral mixing model (LSMM) and Constraint Linear Spectral Mixing Model (CLSMM) in spectral mixing decomposition. The results show that whether using all spectral band data or using the band-selected data, LSMM is in the mixed spectrum. The decomposition effect is better than CLSMM [24]. Luo and Gong simulated the mixed spectrum of soil and vegetation in the Poyang Lake with a mixed model of linear and nonlinear spectra, and the results show that the effect of the linear model is better than that of the nonlinear model. Each of the nonlinear models has its applicable conditions and ranges [25]. Chen and Wang found that atmospheric conditions and terrain factors will affect the decomposition accuracy of the linear spectral mixing model. In small and medium spatial scales with terrain fluctuations, the influence of terrain factors is more significant [26]. Yang et al. measured the laboratory spectra of multiple sets of samples with different materials and component ratios and found that the decomposition accuracy of the linear mixing model is lower when the mixed samples have similar mixing ratios [5]. Similar studies show that the characteristics of spectral reflection of mixed pixels are related to the topological structure of the target and the incident zenith angle. Studies have shown that the more scattered the targets are, the smaller the reflectivity will be, and the larger the incident zenith angle will be, the smaller the reflectivity will be. So considering these two factors will improve the accuracy of mixed pixel decomposition [27].

The spectral characteristics of mixed pixels are the result of a variety of internal and external factors. At present, there are many kinds of research on the factors affecting the decomposition of mixed pixels. Most of them focus on the influence of factors such as structural differences within the pixels, atmospheric scattering, topographic fluctuations, and target roughness on the decomposition accuracy. Nevertheless, the effects of target surface roughness on spectral reflectance and spectral unmixing are often ignored. In order to reveal the effect of roughness on spectral unmixing, other 
factors need to be kept constant during the design of the experiment, and only one factor of roughness is allowed to change. The effect of roughness on the spectral unmixing of mixed pixels was studied. By changing the type of printing paper, four sets of samples with different roughness were designed. Comparing the results of different unmixing with the same roughness and different roughness reveals the effect of surface roughness on the accuracy of spectral unmixing of mixed pixels.

\section{Materials and Methods}

2.1. Sample Design and Spectral Measurement. The ASD FieldSpec3 was used in this experiment, and the probe is equipped with a halogen light source. The inner diameter of the detector is $21 \mathrm{~mm}$, and the outer diameter is $27 \mathrm{~mm}$. Besides, the front field of view angle of the probe is $25^{\circ}$, the waveband range is $350-2500 \mathrm{~nm}$, and the resolution of the measured spectral data is $1 \mathrm{~nm}$ [28]. According to the size of the probe, the design sample diameter is $21 \mathrm{~mm}$, the inner diameter of the black ring is $21 \mathrm{~mm}$, and the outer diameter of the black ring is $27 \mathrm{~mm}$. The sample mixing design method is shown in Figure 1. It is ensured that the probe is aligned with the sample during the spectral measurement. Samples of mixed pixels are printed on four kinds of photo papers: glossy paper, matte paper, frosted paper, and satin paper. The specifications of the four types of photo paper are standard A4 size $(210 * 297 \mathrm{~mm})$, and the weight of each sheet is $13.5 \mathrm{~g}$.

In the laboratory, the endmember spectrum and the mixed pixel spectrum are measured separately. When measuring the spectrum of each sample, the probe is vertically close to the sample and horizontal. Each sample needs to be measured four times at a 90-degree interval; then the spectral processing tool was used to smooth the data. After preprocessing, such as spike correction, the arithmetic average is taken as the original spectral reflectance data for each sample. This experimental study involves spectral data in the visible range. For the impact of the infrared band, the data are resampled from 380 to $780 \mathrm{~nm}$ at $10 \mathrm{~nm}$ intervals. The purpose of resampling the spectral data is to reduce the amount of data and eliminate the data redundancy of adjacent bands by compressing the bands. Doing so will not reduce the method's ability to distinguish the roughness characteristics of mixed pixels. The design diagram of the spectral measurement experiment is shown in Figure 2.

2.2. Linear Spectral Mixing Model. In recent years, the linear spectral mixing model has been widely used in the field of mixed pixel decomposition because it is closer to the remote sensing transmission theory and is convenient and practical. The linear spectral mixing model is to treat the mixed pixel spectrum as the area-weighted linearity of the component spectrum within the pixel. In addition, the model shows that light does not interact between pixel components, and the mixed spectrum is formed after the spectrum of each pixel component enters the sensor. The model assumes that the spectral reflectance of the pixel is the spectral reflection of each endmember inside the pixel. The ratio is a linear combination of weighting coefficients based on its percentage of the pixel area, which is the area-weighted average of the endmember spectrum (equation (1)). Based on LSMM, constraints are added (equation (2)) to form a constrained linear spectral mixture model [23].

$R M S E$ represents the square root of the square sum of the deviation between the observed value and the true value and the ratio of the number of observations. It is used to measure the deviation between the observed value and the actual value. It is used to measure the deviation between the observed value and the actual value. According to the CLSMM model equation, the sample is calculated using the endmember spectral data and the percentage of the pixel area. Regarding the reflectivity and RMSE at each band, RMSE is used to represent the decomposition error of each mixed pixel sample; the equations are as follows:

$$
\begin{aligned}
R_{\lambda} & =\sum_{i=1}^{n} a_{i} f \lambda_{i}+\varepsilon \lambda_{i}, \\
\sum_{i=1}^{n} a_{i} & =1\left(0 \leq a_{i} \leq 1\right), \\
\text { RMSE } & =\sqrt{\frac{1}{m} \sum_{i=1}^{m}\left(R_{i}-R_{i}^{\prime}\right)^{2} .}
\end{aligned}
$$

In equations (1) and (2), $R_{\lambda}$ represents the spectral reflectance of the mixed pixel, $n$ is the number of endmembers, $a_{i}$ is the area percentage of the $i$ th endmembers in the pixel, $f_{\lambda i}$ is the spectral reflectance of the $i$ th endmember in the $\lambda$ band, and $\varepsilon_{\lambda i}$ is the error value. In equation (3), RMSE is the root mean square error of the sample, $m$ represents the number of bands, $R_{i}$ is the calculated reflectivity of the $i$ th sample, and $R_{i}^{\prime}$ represents the measured reflectivity of the $i$ th sample.

2.3. Proportion of White Endmember and Surface Roughness. This article uses the proportion of white endmember ( $P W E$ ) to characterize the mixing ratio of the mixed pixels; that is, the ratio of the white ends to the area of the mixed pixels, expressed in decimals:

$$
\mathrm{PWE}=\frac{A W}{A W+A B} .
$$

In equation (4), $P W E$ represents the mixing index of the sample, $A_{B}$ represents the area of the black endmembers in the sample, $A w$ represents the area of the white endmembers, $P W E \in[0,1], 0$ represents pure black pixels, and 1 represents pure white pixels.

Surface roughness is represented by $R a$, which refers to the small spacing and the unevenness of tiny peaks and valleys on the surface of an object. The smaller the surface roughness, the smoother the surface. The arithmetic mean deviation of the contour is the arithmetic mean of the absolute deviation of the contour within the sampled length. In other words, it is the arithmetic means of the absolute value of the distance from each point on the surface contour to the midline of the contour within the 


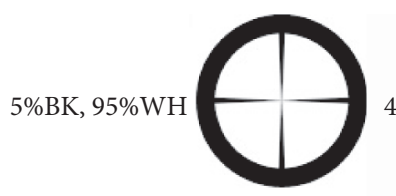

$40 \% \mathrm{BK}, 60 \% \mathrm{WH}$
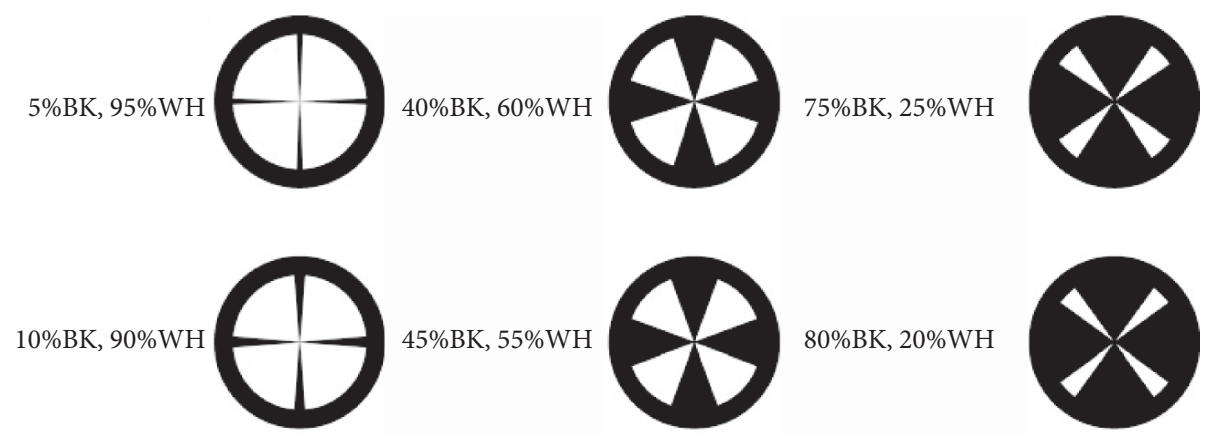

$45 \% \mathrm{BK}, 55 \% \mathrm{WH}$

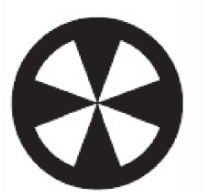

$80 \% \mathrm{BK}, 20 \% \mathrm{WH}$
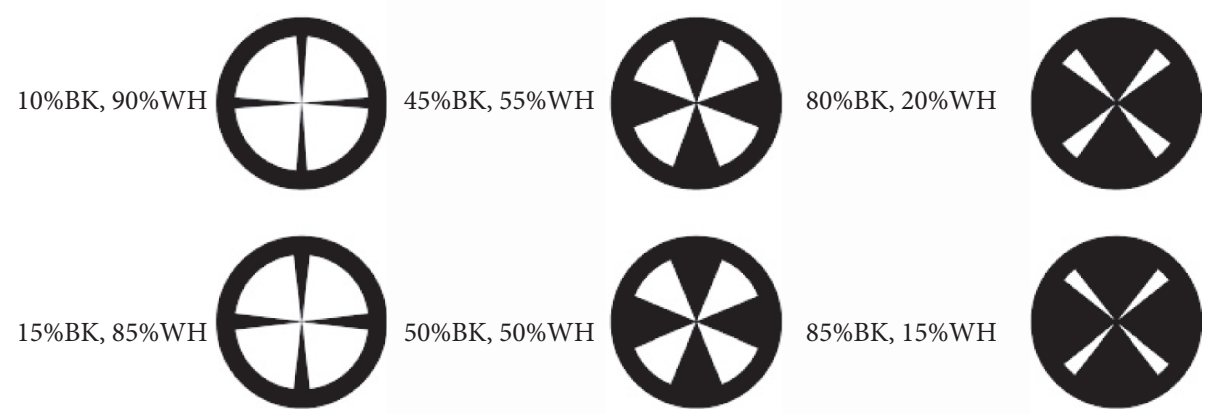

$50 \% \mathrm{BK}, 50 \% \mathrm{WH}$

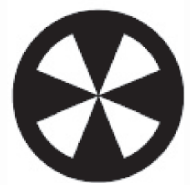

$85 \% \mathrm{BK}, 15 \% \mathrm{WH}$
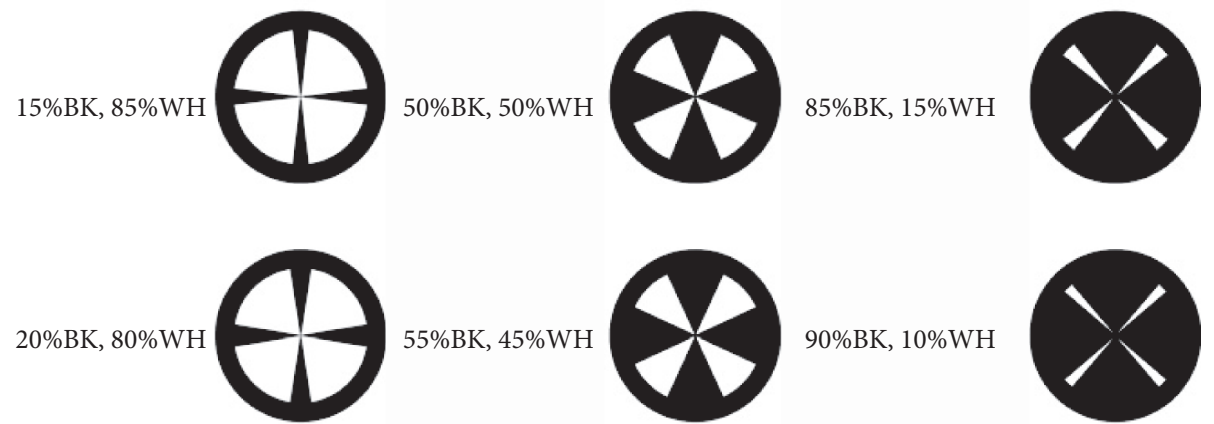

$90 \% \mathrm{BK}, 10 \% \mathrm{WH}$
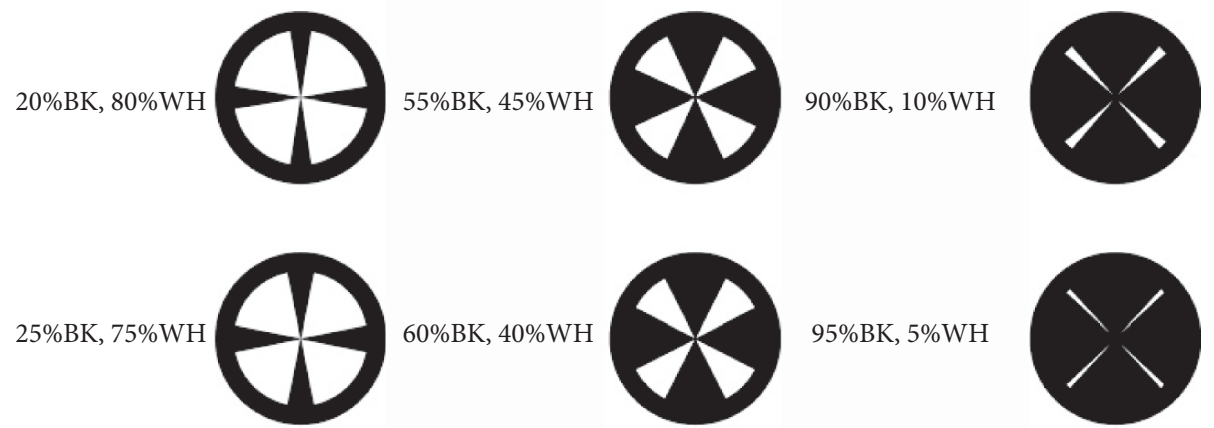

$60 \% \mathrm{BK}, 40 \% \mathrm{WH}$

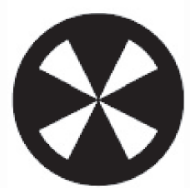

95\%BK, 5\%WH
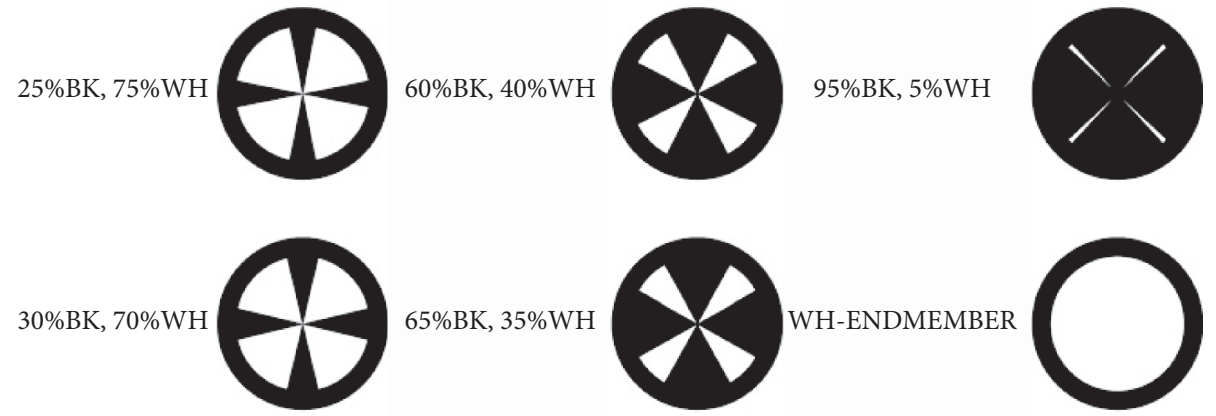

WH-ENDMEMBER
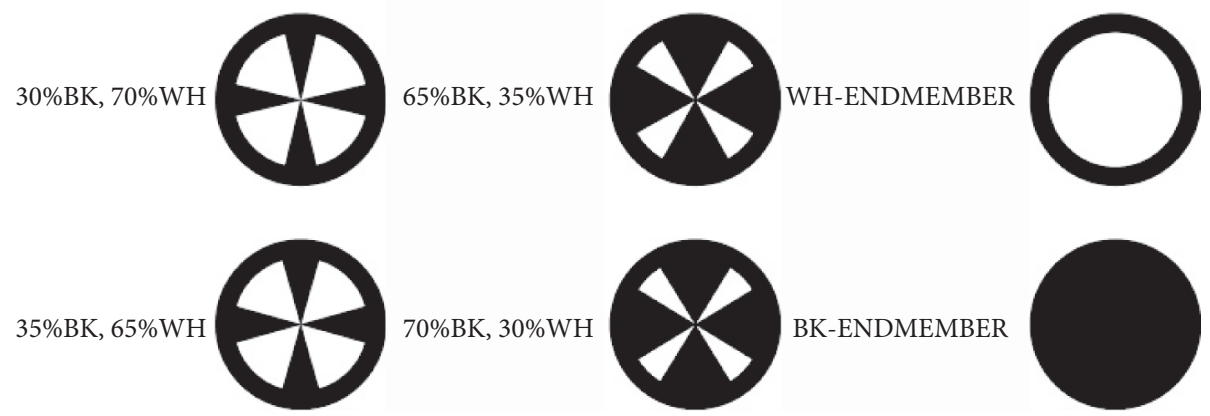

$70 \% \mathrm{BK}, 30 \% \mathrm{WH}$

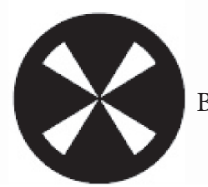

BK-ENDMEMBER

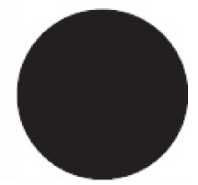

FIgURe 1: Sample design of mixed pixels.

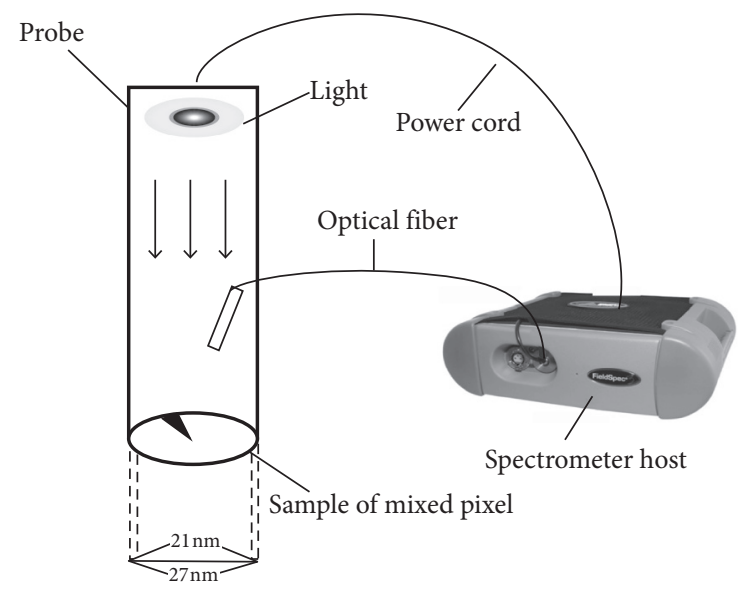

Figure 2: The experimental design of spectral measurement. sample length. In the actual measurement, the more the number of measurement points, the more accurate $R a$. The surface roughness test of this test sample uses a handheld high-precision roughness meter (Jitech TR200). The instrument gives the parameters which are not only in compliance with Chinese standards (China GB/T 60622002) but also compatible with ISO, DIN, ANSI, JIS, US, German, Japanese, and British national standards. The instrument is suitable for metal and nonmetal tested parts with rough surfaces. The degree of measurement is not affected by high temperature and high humidity working environment. The measurement range of the roughness meter is $0.005-16.00 \mu \mathrm{m}$, the measurement accuracy is $0.001 \mu \mathrm{m}$, and the measurement error is $\leq \pm 7 \%$. Surface roughness test points at different photo papers. The numbering design is shown in Figure 3. 


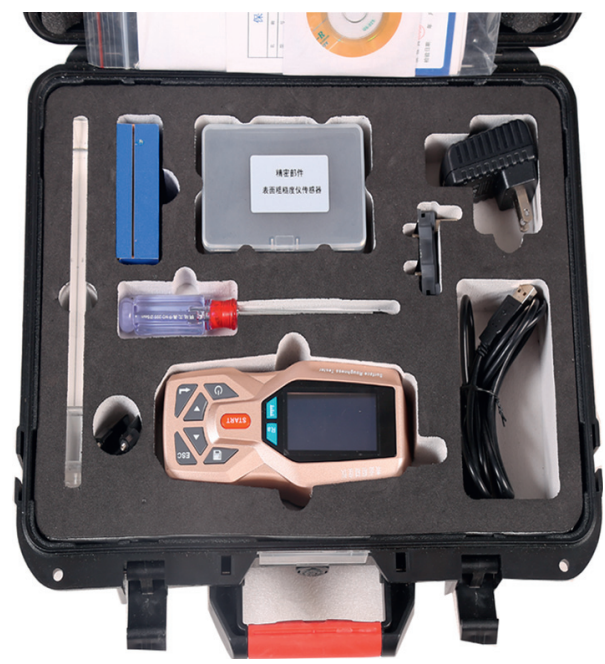

(a)

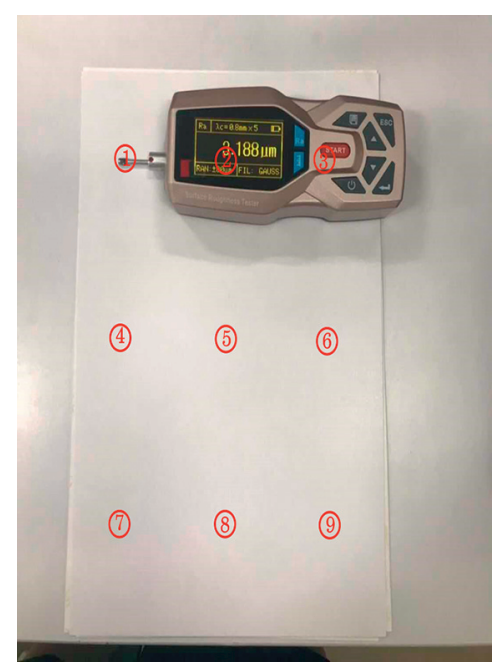

(b)

Figure 3: Surface roughness meter (a) and test number design (b).

\section{Results and Discussion}

3.1. Influence of Mixing Ratio and Surface Roughness on the Reflectivity of Mixed Pixels. In order to reveal the influence of mixing ratio and surface roughness on the reflectivity of mixed pixels, the black and white endmembers and the reflectance data of three typical mixed pixel samples are $P W E=0.8,0.5$, and 0.2 . These samples were selected for comparative analysis. It can be seen from Figures 4(a)-4(d) that the reflectance of the white endmembers on the satin paper and matte paper is slightly higher than one at $440-450 \mathrm{~nm}$. The phenomenon is caused by the Rayleigh scattering, but the small overflow amplitude will not affect the subsequent analysis results. Therefore, there is no need to modify it. The reflectance of the three typical mixed pixels gradually decreases with the decrease in the proportion of white endmembers. The three groups of spectral curves are evenly spaced, indicating that the effect of the experimental data better reflects the effect of the mixing ratio. Regarding reflectivity difference, further comparison shows that when the white area is large, the reflectance of the same mixed pixel on different photo papers varies greatly. This difference is particularly significant in the trough region of $550-600 \mathrm{~nm}$. When $P W E=0.8$, by mixing, the reflectivity of the pixels from high to low is satin paper (blue) $>$ matte paper (green) $>$ matte paper (red) $>$ glossy paper (black). As the proportion of white in the mixed pixel decreases, the difference in reflectance of mixed pixels of different photo papers gradually decreases. When $P W E=0.1$, when the peaks and troughs of the reflectance spectrum disappear, the spectral curve appears as a straight line close to 0 (380-700 $\mathrm{nm}$ band interval). When the proportion of white endmembers in the mixed pixel is close to 0 , there is a reflection on the four kinds of photo paper. The rate curves are the same, indicating that the difference in reflectance caused by roughness at this time is negligible.

In order to quantitatively reveal the difference in reflectance of different photo paper mixed pixels caused by the change in the ratio of white endmembers, the average of the reflectance of all mixed pixels in the visible light band of $380-780 \mathrm{~nm}$ was statistically calculated (Table 1). The reflectivity of the mixed pixel on four kinds of paper is divided into four groups. Moreover, the absolute value of the range within the group is calculated, which can reflect the difference of reflectivity. As can be seen from the table, the average reflectance of the satin paper with large surface roughness is higher, and the roughness is small. The reflectivity of glossy paper is low. This trend is not apparent on some mixed pixels, which is mainly because the data of the $380-440 \mathrm{~nm}$ and $700-780 \mathrm{~nm}$ bands are included in the statistical average, and the two band ranges are affected by Rayleigh scattering and near-infrared effects. Although the difference will be lost, the integrity of the visible light data is retained to facilitate the analysis of the spectrum change law.

As can be seen from Figure 5, the reflectance range decreases with the decrease in the proportion of white endmember, and this trend is undeniable. To further analyze the change rule, we perform a Pearson correlation analysis of the white area ratio and the reflectance range and find that there are significant correlation characteristics and the correlation coefficient $r$ is 0.796 . The significance test Sig is less than 0.01 , which is a significant positive correlation. We perform a function fit on the two and find that the linear equation $y=a * x+b$ has the best fitting effect and $R^{2}$ reaches 0.634 . In summary, we found that the reflectivity of mixed pixels in different photo papers will vary due to the surface roughness of the photo paper. This difference in reflectivity is closely related to the PWE of mixed pixels. There are some differences between pure black endmembers and pure white endmembers on different photo papers and whether the surface roughness will lead to the accuracy of linear spectral unmixing needs to be further verified by error contrast experiments.

3.2. Analysis of Test Data for Surface Roughness of Four Kinds of Photo Paper. Use a surface roughness tester to measure 


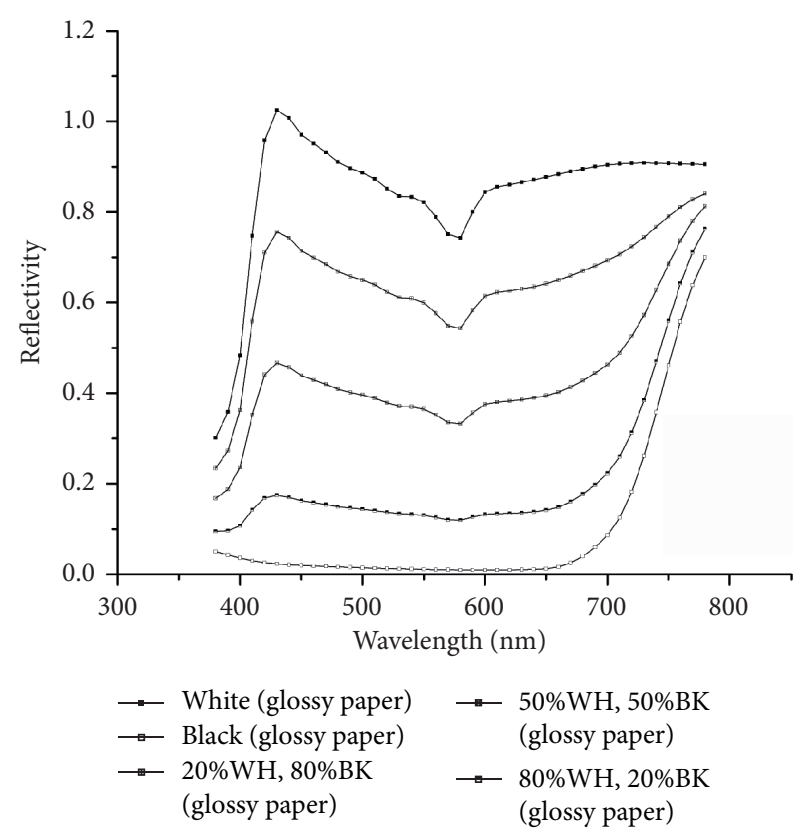

(a)

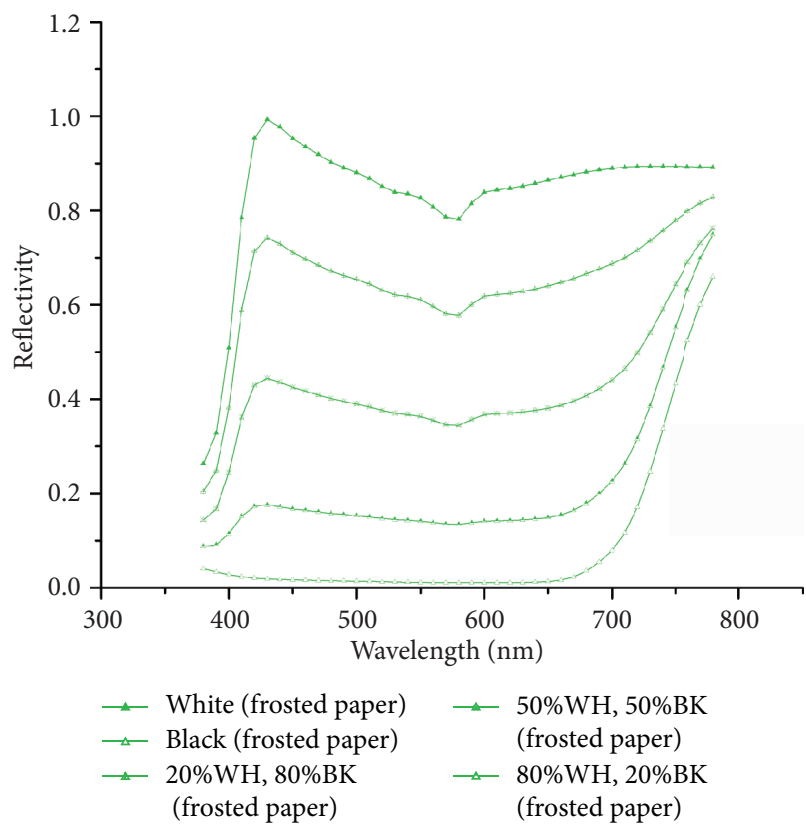

(c)

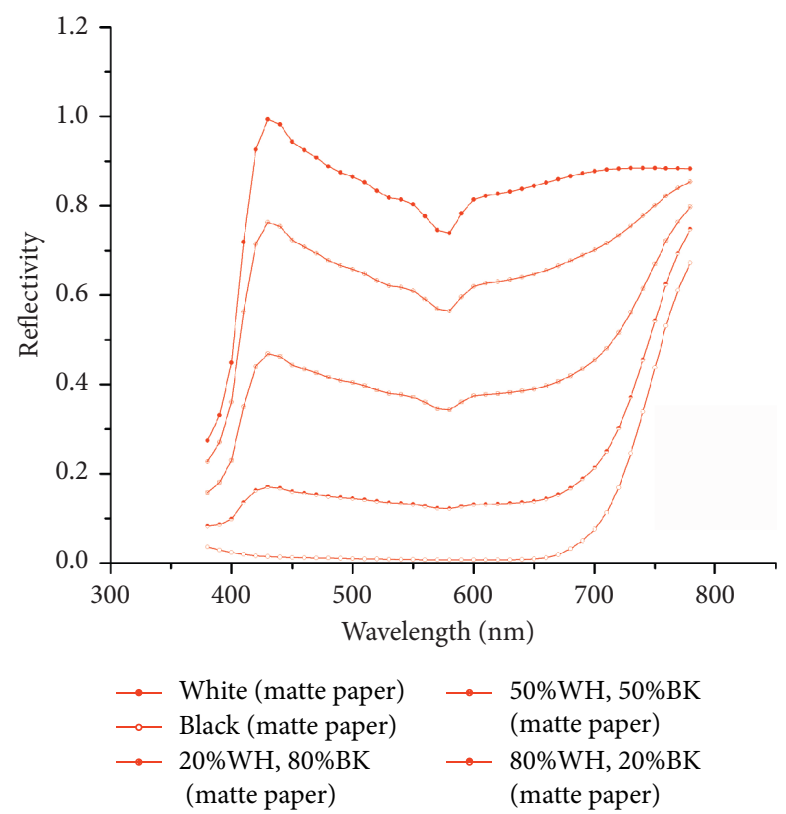

(b)

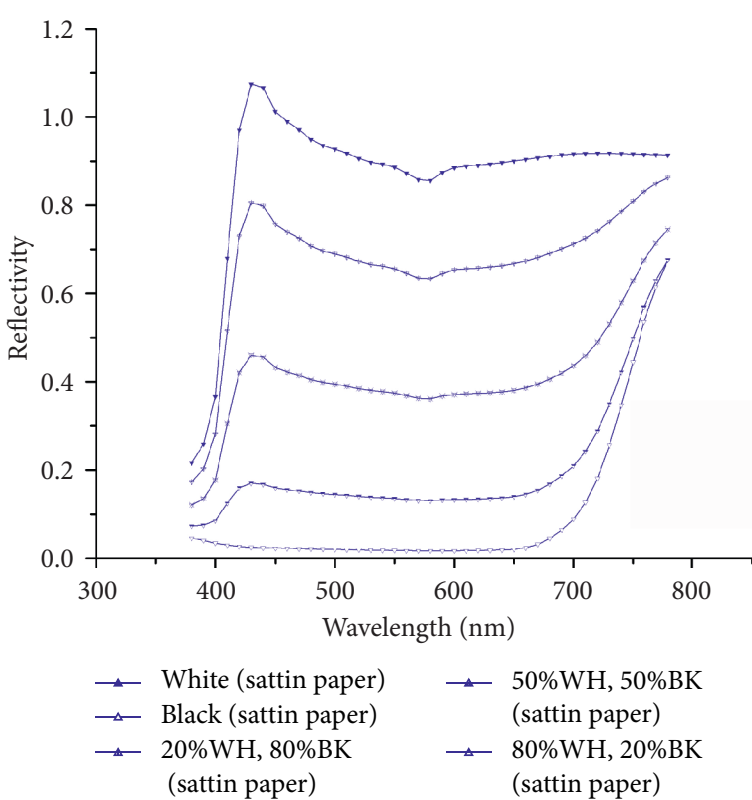

(d)

FIGURE 4: Reflectance spectra of mixed pixels on different papers.

the roughness of four kinds of photo paper: glossy paper, matte paper, matte paper, and satin paper. Nine test points were selected for each photo paper. The roughness measurement data of 9 test points were counted, and the roughness of each paper was expressed by arithmetic mean value. The specific results of the roughness of the photo paper are shown in the following table. From Table 2, the test results of the three kinds of photo paper can be seen: glossy paper, suede paper, and matte paper are ideal. The difference is small. The satin paper has roughness data measured at only 3 of the 9 test points, and the difference is significant. The remaining 6 points show out of range. It is speculated that this may be due to glossy paper or suede paper. The surface texture particles of sandpaper and matte paper are relatively evenly distributed. In contrast, the satin paper is unevenly distributed, which causes the roughness to exceed the measuring range of the instrument in most cases.

3.3. Analysis of the Influence of Surface Roughness on Linear Spectral Unmixing of Mixed Pixels. The linear spectral mixing model is used to unmix all the mixed pixels, and the actual theoretical reflectance and the observed reflectance are calculated to obtain the root mean square error RMSE. 
TABLE 1: Average reflectance statistics of mixed pixels on four kinds of photo paper.

\begin{tabular}{|c|c|c|c|c|c|}
\hline$P W E$ & Glossy paper & Matte paper & Frosted paper & Satin paper & Range of reflectivity \\
\hline 1.000 & 0.844 & 0.820 & 0.838 & 0.869 & 0.049 \\
\hline 0.950 & 0.770 & 0.779 & 0.768 & 0.804 & 0.036 \\
\hline 0.900 & 0.726 & 0.734 & 0.725 & 0.755 & 0.030 \\
\hline 0.850 & 0.685 & 0.692 & 0.685 & 0.713 & 0.027 \\
\hline 0.800 & 0.643 & 0.652 & 0.644 & 0.671 & 0.027 \\
\hline 0.750 & 0.602 & 0.611 & 0.612 & 0.608 & 0.010 \\
\hline 0.700 & 0.561 & 0.587 & 0.572 & 0.572 & 0.026 \\
\hline 0.650 & 0.523 & 0.546 & 0.531 & 0.529 & 0.023 \\
\hline 0.600 & 0.488 & 0.510 & 0.494 & 0.495 & 0.022 \\
\hline 0.550 & 0.471 & 0.469 & 0.459 & 0.458 & 0.013 \\
\hline 0.500 & 0.433 & 0.431 & 0.418 & 0.415 & 0.017 \\
\hline 0.450 & 0.396 & 0.378 & 0.402 & 0.381 & 0.024 \\
\hline 0.400 & 0.359 & 0.340 & 0.361 & 0.343 & 0.021 \\
\hline 0.350 & 0.322 & 0.303 & 0.323 & 0.307 & 0.020 \\
\hline 0.300 & 0.285 & 0.269 & 0.287 & 0.271 & 0.018 \\
\hline 0.250 & 0.250 & 0.248 & 0.250 & 0.236 & 0.015 \\
\hline 0.200 & 0.216 & 0.211 & 0.220 & 0.203 & 0.017 \\
\hline 0.150 & 0.181 & 0.177 & 0.185 & 0.171 & 0.013 \\
\hline 0.100 & 0.148 & 0.142 & 0.149 & 0.139 & 0.010 \\
\hline 0.050 & 0.118 & 0.113 & 0.118 & 0.111 & 0.008 \\
\hline 0.000 & 0.097 & 0.089 & 0.091 & 0.099 & 0.010 \\
\hline
\end{tabular}

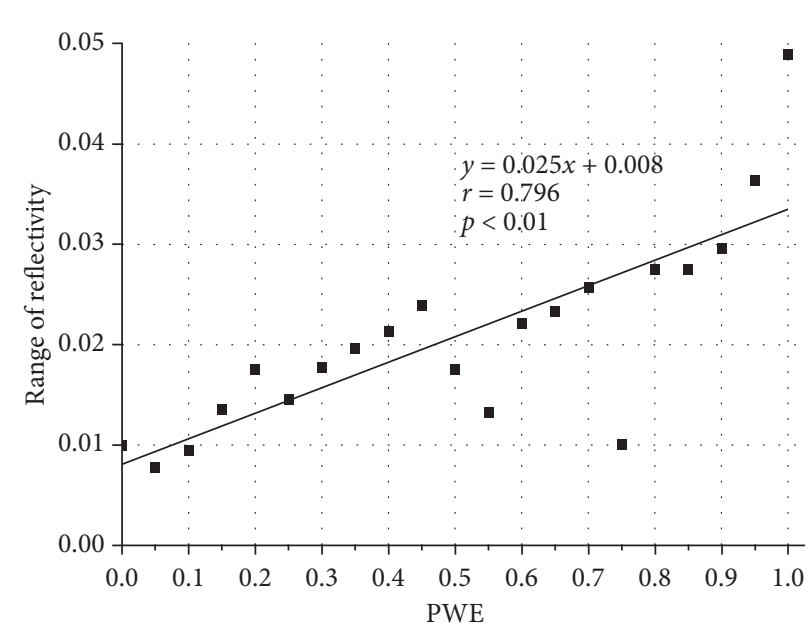

FIGURE 5: Relationship between the ratio of white endmembers and the extreme reflectance.

TABLE 2: Statistical table of roughness test data (unit: $\mu \mathrm{m}$ ).

\begin{tabular}{lcccccccccc}
\hline \multirow{2}{*}{ Paper type } & \multicolumn{10}{c}{ Number of test points } \\
& 1 & 2 & 3 & 4 & 5 & 6 & 7 & 8 & 9 & Average roughness \\
\hline Glossy paper & 0.152 & 0.135 & 0.157 & 0.168 & 0.121 & 0.119 & 0.194 & 0.137 & 0.171 & 0.150 \\
Matte paper & 1.398 & 1.283 & 1.302 & 1.459 & 1.249 & 1.134 & 1.484 & 1.309 & 1.264 & 1.320 \\
Frosted paper & 3.188 & 3.626 & 3.192 & 3.369 & 3.317 & 3.915 & 3.091 & 3.046 & 3.309 & 3.339 \\
Satin paper & 4.524 & No to scale & No to scale & 9.216 & No to scale & No to scale & 6.791 & No to scale No to scale & - \\
\hline
\end{tabular}

The changes of the RMSE can intuitively reflect the effect of the linear unmixing of the mixed pixels. It can be seen from Figure 6 that the distribution of the unmixing error of the mixed pixels on each type of photo paper shows a parabolic function shape. With $P W E=0.5$ being the symmetry of the axial direction, which gradually decreases on both sides, when $P W E$ of the mixed pixel is 0.4 , at 0.6 , the unmixing error is more significant. When the $P W E$ equals 0.05 or 0.95 , the unmixing error is the smallest. The unmixing error of the mixed pixels on the satin paper is the largest, and the 


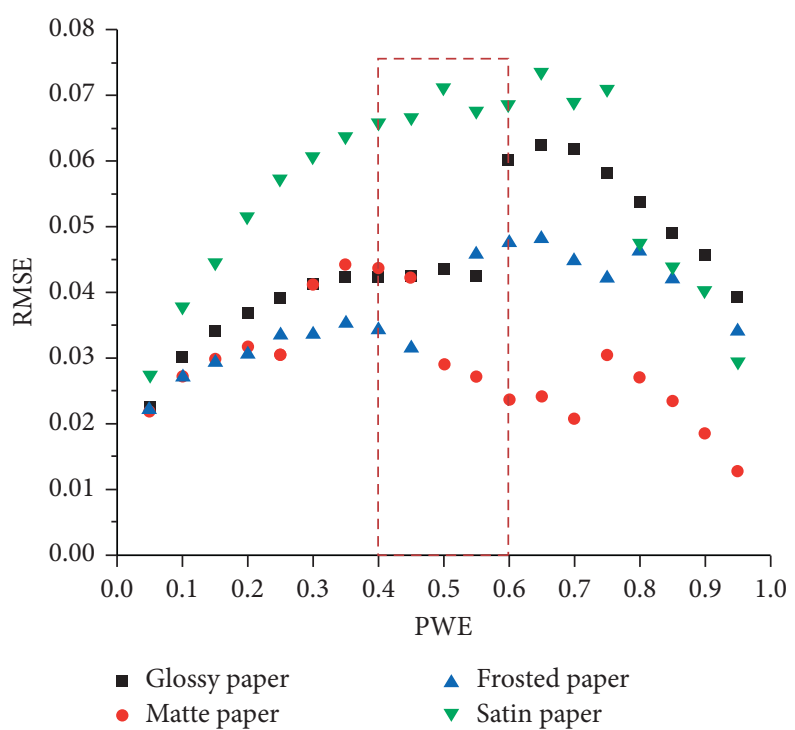

FIGURE 6: Relationship between the proportion of white endmembers on different photo papers and the RMSE of the unmixing error.

TABLE 3: Statistics of mixed pixel linear unmixing errors.

\begin{tabular}{|c|c|c|c|c|c|}
\hline \multicolumn{6}{|c|}{ Paper type } \\
\hline$P W E$ & Glossy paper (\%) & Matte paper (\%) & Frosted paper (\%) & Satin paper $(\%)$ & Average error (\%) \\
\hline 0.95 & 0.20 & 0.02 & 0.15 & 0.11 & 0.12 \\
\hline 0.9 & 0.29 & 0.05 & 0.21 & 0.22 & 0.19 \\
\hline 0.85 & 0.35 & 0.08 & 0.26 & 0.27 & 0.24 \\
\hline 0.8 & 0.45 & 0.11 & 0.34 & 0.34 & 0.31 \\
\hline 0.75 & 0.56 & 0.15 & 0.29 & 0.83 & 0.46 \\
\hline 0.7 & 0.68 & 0.07 & 0.35 & 0.83 & 0.48 \\
\hline 0.65 & 0.75 & 0.11 & 0.44 & 1.03 & 0.58 \\
\hline 0.6 & 0.74 & 0.11 & 0.46 & 0.95 & 0.56 \\
\hline 0.55 & 0.38 & 0.16 & 0.46 & 0.99 & 0.50 \\
\hline 0.5 & 0.44 & 0.20 & 0.58 & 1.22 & 0.61 \\
\hline 0.45 & 0.46 & 0.47 & 0.25 & 1.16 & 0.59 \\
\hline 0.4 & 0.50 & 0.56 & 0.33 & 1.26 & 0.66 \\
\hline 0.35 & 0.56 & 0.65 & 0.39 & 1.32 & 0.73 \\
\hline 0.3 & 0.60 & 0.63 & 0.39 & 1.35 & 0.74 \\
\hline 0.25 & 0.61 & 0.38 & 0.45 & 1.39 & 0.71 \\
\hline 0.2 & 0.63 & 0.47 & 0.42 & 1.31 & 0.71 \\
\hline 0.15 & 0.65 & 0.51 & 0.46 & 1.16 & 0.69 \\
\hline 0.1 & 0.61 & 0.52 & 0.49 & 1.03 & 0.66 \\
\hline 0.05 & 0.43 & 0.43 & 0.42 & 0.68 & 0.49 \\
\hline Average error & 0.52 & 0.30 & 0.38 & 0.92 & 0.53 \\
\hline
\end{tabular}

unmixing error of the mixed pixels on the suede paper is the smallest. However, on the whole, the mixed pixel unmixing error on the four kinds of roughness photo paper is less than 0.08 , and in some cases, the unmixing error is 0.01 , and the average unmixing error of 76 mixed pixels is 0.042 . According to statistical principles, it can be known that for the prediction of continuous variables, we can measure it with the help of RMSE mean square error. When the RMSE is smaller, the result means that the model works better. In other words, the predicted value will be closer to the actual value. When RMSE approaches 0 , the surface roughness has little effect on the resolution accuracy of mixed pixels.

The influence of surface roughness on the unmixing accuracy of the linear spectrum was further explored, and the average unmixing error, average reflectance, and unmixing error in the band range of $380-780 \mathrm{~nm}$ were calculated. The difference can be seen in Table 3 . The error of the unmixing of mixed pixels on the same type of photographic paper is $0.92 \%$, and the maximum error of unmixing of the same type of mixed pixels on different photographic papers is $0.69 \%$. The average unmixing error of all mixed pixels is only spectral reflectance measurement error of $0.53 \%$, less than $1 \%$. Experimental results show that although the four kinds of photo papers with different surface roughness have different spectral reflectance, they will not affect the results of linear spectral unmixing. Combined with the roughness data, when the surface roughness $R a$ of the test sample is less than $3.339 \mu \mathrm{m}$, use a spectrometer to 
perform the spectral measurement and perform a linear spectral unmixing experiment. The effect of roughness on the unmixing accuracy can be ignored.

According to the basic rules of ground object radiation and reflection, the phenomenon of photon energy reflected by the object surface includes specular reflection and diffuse reflection, which is related to the roughness of the object surface. This phenomenon is related to the roughness of the surface of the measured object. When the surface roughness is less than the human wave, spectral reflection is formed when the wavelength is 5\%, and the reflection angle is equal to the angle of incidence at this time. When the surface roughness is greater than the wavelength of the incident wave, diffuse reflection is formed. The reflection line is not unique and irregular. The structural complexity of the mixed pixel itself and the external changes in the environment lead to different situations in which light is scattered under different experimental scenarios, so it is particularly important to choose different models according to the actual situation. Studies show that, under stable light conditions and smooth surfaces, the waveband was received by the detector. The signal is mainly single-scattering. At this time, the linear spectrum mixing model has a better decomposition effect. When the surface of the pixel is rough or fluctuates, the multiple scattering effects between the internal components of the pixel are intensified. The model is more in line with the actual situation. Therefore, the influence factor of multiple scattering in the pixel is the key to model selection and unmixing accuracy. The experiment uses linear spectral mixing. The error between the results obtained by the linear spectral mixing model and the actual situation is less than $1 \%$. The experimental results show that if the surface roughness of the measured sample is less than the maximum wavelength of the spectrometer, the photon energy on the surface of the measured object is mainly specular reflection, with almost no diffuse reflection. At this time, it is still an excellent choice to decompose the mixed pixel by linear spectral mixing model.

\section{Conclusion}

The papers with different surface roughness have a specific effect on the reflectivity of the mixed pixels. This phenomenon is more apparent when the proportion of white endmember $(P W E)$ is significant, and as the white area ratio decreases, the reflectance difference gradually decreases. When the mixed pixels are close to the pure black endmembers, the reflectance of the mixed pixels on different photo papers is the same. When the surface roughness $R a$ of the sample is less than $3.339 \mu \mathrm{m}$, the mixed pixel decomposition experiment is carried out in the range of visible light band using the linear spectral mixing model. The average unmixing error of the mixed pixel with different roughness is no more than $0.53 \%$, which is less than $1 \%$ of the standard spectral measurement error. When the surface roughness of the sample is controlled within a specific range, the effect of roughness on the unmixing accuracy of the mixed pixels is small, and this effect can be almost ignored. When the ASD FieldSpec3 spectrometer is used for spectral reflectance measurement and linear unmixing experiment, multiple scattering in the image element is the key to model selection and unmixing accuracy. If the surface roughness of the sample under test is less than the maximum wavelength of the spectrometer, the photon energy on the surface of the object is mainly specular reflection, and diffuse reflection hardly occurs. At this time, it is still an excellent choice to decompose the mixed pixel by linear spectral mixing model.

\section{Data Availability}

The data used to support the findings of this study are available from the corresponding author upon request after this project has been finished in 2021.

\section{Conflicts of Interest}

The authors declare that there are no conflicts of interest regarding the publication of this article.

\section{Acknowledgments}

The study was supported by the remote sensing geochemistry disciplinary innovation team, Kunming University of Science and Technology, Kunming, China. Furthermore, the work was funded by the National Natural Science Fund of China (41101343) and 2018 Yunnan Province Ph.D. Academic Newcomer Award.

\section{References}

[1] N. Keshava and J. F. Mustard, "Spectral unmixing," IEEE Signal Processing Magazine, vol. 19, no. 1, pp. 44-57, 2002.

[2] M. Khodadadzadeh, J. Li, A. Plaza, H. Ghassemian, and J. M. Bioucas-Dias, "Spectral-spatial classification of hyperspectral data using local and global probabilities for mixed pixel characterization," IEEE Transactions on Geoscience and Remote Sensing, vol. 52, no. 10, pp. 6298-6314, 2014.

[3] P. F. Hsieh, L. C. Lee, and N. Y. Chen, "Effect of spatial resolution on classification errors of pure and mixed pixels in remote sensing," IEEE Transactions on Geoscience and Remote Sensing, vol. 39, no. 12, pp. 2657-2663, 2002.

[4] J. Lan, J. Zou, Y. Hao et al., "Research progress on unmixing of hyperspectral remote sensing imagery," Journal of Remote Sensing, vol. 22, no. 1, pp. 13-27, 2018, in Chinese.

[5] S.-H. Yang, K.-H. Kim, Y. K. Park, and S.-G. Lee, "Error analysis and compensation for the volumetric errors of a vertical machining centre using a hemispherical helix ball bar test," The International Journal of Advanced Manufacturing Technology, vol. 23, no. 7-8, pp. 495-500, 2004.

[6] J. Chen, L. Ma, X. Chen et al., "Research progress of spectral mixture analysis," Journal of Remote Sensing, vol. 20, no. 5, pp. 1102-1109, 2016, in Chinese.

[7] J. A. Desanto, G. S. Brown, and I. Brown, "I analytical techniques for multiple scattering from rough surfaces," Progress in Optics, vol. XXIII, pp. 1-62, 1986.

[8] X. Shao, C. Cao, and T.-C. Liu, "Spectral dependent degradation of the solar diffuser on suomi-NPP VIIRS due to surface roughness-induced Rayleigh scattering," Remote Sensing, vol. 8, no. 3, p. 254, 2016. 
[9] D. Schröder and A. Pohl, "Modeling (non-)uniform scattering distributions in geometrical acoustics," The Journal of the Acoustical Society of America, vol. 133, no. 5, p. 3530, 2013.

[10] E. Larose, T. Planes, V. Rossetto et al., "Locating a small change in a multiple scattering environment," Applied Physicsletters, vol. 96, no. 20, pp. 204101.1-204101.3, 2010.

[11] J. Cierniewski, "A model for soil surface roughness influence on the spectral response of bare soils in the visible and nearinfrared range," Remote Sensing of Environment, vol. 23, no. 1, pp. 97-115, 1987.

[12] U. Persson, "Measurement of surface roughness on rough machined surfaces using spectral speckle correlation and image analysis," Wear, vol. 160, no. 2, pp. 221-225, 1993.

[13] D. Prazak and M. Ohlidal, "Laser speckle spectral correlation and surface roughness," Proceedings of Spie the International Society for Optical Engineering, vol. 4356, pp. 339-346, 2001.

[14] V. Cotoni and A. Le Bot, "Specular and diffuse reflections of rays in coupled thin plates at high frequencies," Journal of Sound \& Vibration, vol. 265, no. 1, pp. 23-41, 2003.

[15] R. V. Morris, S. C. Neely, and W. W. Mendell, “Application of Kubelka-Munk theory of diffuse reflectance to geologic problems: the role of scattering," Geophysical Research Letters, vol. 9, no. 2, pp. 113-116, 1982.

[16] S. Morimine, T. S. Norimoto, and T. Hasegawa, "Surface selection rule of infrared diffuse reflection spectrometry for analysis of molecular adsorbates on a rough surface of a nonabsorbing medium," Analytical Chemistry, vol. 86, no. 9, pp. 4202-4208, 2014.

[17] M. Xie, X.-L. Mu, and X. Xia, "Bi-directional reflectance properties of granite surface based on rough topographic data," Journal of Infrared and Millimeter Waves, vol. 28, no. 2, pp. 119-123, 2009, in Chinese.

[18] Y. Lu and Y. Zhao, "Study of retrieving the sandy surface roughness land based on the bidirectional reflectance model," Spectroscopy \& Spectral Analysis, vol. 35, no. 11, pp. 31233128, 2015, in Chinese.

[19] Y. Kang, J. Wang, H. Zhou et al., "Soil surface roughness estimation using multiangular remote sensing observations: a preliminary study," Journal of Remote Sensing, vol. 17, no. 1, pp. 180-192, 2013, in Chinese.

[20] M. Pan, H.-X. Qi, G.-H. Xiao, and R. Shu, "Study of mixed pixel linear unmixing model based on high accurate endmember," Journal of Infrared \& Millimeter Waves, vol. 29, no. 5, pp. 357-361, 2010.

[21] A. Mecocci, "Theoretical and experimental assessment of noise effects on least-squares spectral unmixing of hyperspectral images," Optical Engineering, vol. 44, no. 8, Article ID 087008, 2005.

[22] L. Drumetz, J. Chanussot, and C. Jutten, "Spectral unmixing: a derivation of the extended linear mixing model from the hapke model," IEEE Geoscience and Remote Sensing Letters, vol. 99, pp. 1-5, 2019.

[23] R. Heylen and P. Gader, "Nonlinear spectral unmixing with a linear mixture of intimate mixtures model," IEEE Geoscience and Remote Sensing Letters, vol. 11, no. 7, pp. 1195-1199, 2014.

[24] Q. Wang, Q. Lin, M. Li et al., "Comparison of two spectral mixture analysis models," Spectroscopy and Spectral Analysis, vol. 29, no. 10, pp. 2602-2605, 2009, in Chinese.

[25] H. Luo and J. Gong, "Effects of linear and nonlinear spectra mixing models on simulating mixing spectra of soil and canopy," Bulletin of Surveying and Mapping, vol. 5, pp. 6-10, 2005, in Chinese.
[26] S. Chen and T. Wang, "Study on uncertainty of environment factors relating to linear spectral mixing model," Geo-information Science, vol. 9, no. 6, pp. 49-53, 2007, in Chinese.

[27] G. Yan, Y. Zhao, Y. Ning et al., "Study on the main influencing factors of mixed-pixel spectral characteristics," Spectroscopy and Spectral Analysis, vol. 29, no. 12, pp. 3358-3361, 2009, in Chinese.

[28] L. Martin, M. Wernand, A. Hommersom et al., "Intercomparison in the field between the new WISP-3 and other radiometers (TriOS Ramses, ASD FieldSpec, and TACCS)," Journal of Applied Remote Sensing, vol. 6, no. 1, Article ID 063615, 2012. 